\title{
Charge Qubit Purification by an Electronic Feedback Loop
}

\author{
Gerold Kießlich $*$ Gernot Schaller, Clive Emary, and Tobias Brandes \\ Institut für Theoretische Physik, Hardenbergstraße 36, \\ Technische Universität Berlin, D-10623 Berlin, Germany
}

\begin{abstract}
We propose the manipulation of an isolated qubit by a simple instantaneous closed-loop feedback scheme in which a time-dependent electronic detector current is directly back-coupled into qubit parameters. As specific detector model we employ a capacitively coupled single-electron transistor. We demonstrate the stabilization of pure delocalized qubit states above a critical detector-qubit coupling. This electronic purification is independent of the initial qubit state and is accomplished after few electron jumps through the detector. Our simple scheme can be used for the efficient and robust initialization of solid-state qubits in quantum computational algorithms at arbitrary temperatures.

PACS numbers: 03.65.Ta, 05.40.-a, 73.23.-b, 85.35.-p
\end{abstract}

The ability to measure and control individual quantum systems (e.g. qubits) is a major requirement for contemporary and future information technologies [1]. The measurement process - the contact between classical measurement device and nano-scale object - plays a key role in controlling quantum systems and mostly introduces a source of decoherence. State-of-the-art measurement technologies in solid-state environments mainly rely on the change of current flow through an on-chip detector due to Coulomb repulsion with the charges in the measured system (see e.g. Refs. 2]). The continuous monitoring of charge qubits by such charge detectors yields relaxation and decoherence as studied experimentally e.g. in Ref. [3] and theoretically in Refs. [4]. However, the detector signal contains detailed information on the qubit dynamics which can be used for its control.

An elegant control strategy, which has been successfully applied in a wide variety of physical and engineering systems [5], is stabilization of favored system states due to feedback. For quantum systems such as qubits, several theoretical proposals to enhance quantum coherence by feedback have been made. These are mainly based upon sophisticated techniques, e.g., the on-chip conversion of detector outputs into qubit density matrix evolutions [6, 7] or monitoring the phase of qubit oscillations by detector current quadratures [8]. However, a much simpler scheme was recently used in the first experimental use of feedback to increase quantum coherence of a spin qubit [9].

Here, we study a charge qubit consisting of a single electron confined in a two-state system which is continuously monitored by the current through a single-electron transistor (SET) [see Fig. 1. The SET current will be directly fed back into qubit parameters which allow the stabilization of arbitrary pure qubit states.

Model. - The SET electronic level is shifted depending on where the electron is located in the two-level system due to Coulomb interaction. Moreover, the charge qubit electron is assumed to be coupled to thermal phonons of the surrounding matrix material. The full Hamiltonian reads

$$
\begin{aligned}
H & =H_{\mathrm{SET}}+H_{\mathrm{q}}+\frac{1}{2} d^{\dagger} d\left[U \hat{\sigma}_{z}+\left(U_{t}+U_{b}\right) \mathbb{1}\right] \\
H_{\mathrm{q}} & =\frac{1}{2} \varepsilon \hat{\sigma}_{z}+T_{c} \hat{\sigma}_{x}+\sum_{Q}\left[\frac{1}{2} \hat{\sigma}_{z} g_{Q} A_{Q}+\Omega_{Q} a_{Q}^{\dagger} a_{Q}\right],
\end{aligned}
$$

with $\varepsilon \equiv \varepsilon_{t}-\varepsilon_{b}$ and $A_{Q} \equiv a_{-Q}+a_{Q}^{\dagger}$. The SET with Hamiltonian $H_{\mathrm{SET}}=\varepsilon_{d} d^{\dagger} d+\sum_{k a=L, R}\left[\varepsilon_{k a} c_{k a}^{\dagger} c_{k a}+\right.$ $\left(t_{k a} c_{k a} d^{\dagger}+\right.$ h.c. $\left.)\right]$ contains a single electronic level (with creation/annihilation operators $d^{\dagger} / d$ ) which is electrostatically coupled to the charge qubit levels by $U \equiv U_{t}-U_{b} \geq 0$. The charge qubit Hamiltonian is given in the Bloch representation with Pauli matrices $\hat{\sigma}_{z}=|t\rangle\langle t|-| b\rangle\langle b|$ and $\hat{\sigma}_{x}=|t\rangle\langle b|+| b\rangle\langle t|$. The phononic modes with wave vector $Q$ and energy $\Omega_{Q}$ are diagonally coupled to the qubit assuming weak coupling $T_{c}$.

Equations of Motion. - The standard Born-Markovsecular approximation yields a quantum master equation $\dot{\rho}(t)=\mathcal{L} \rho(t)$ for the system (SET + qubit) density matrix $\rho(t)$ with the Liouvillian superoperator $\mathcal{L}$ where the SET leads and the phonon environment are treated in thermal equilibrium. Due to the secular approximation the 4 populations and 2 coherences contained in $\rho(t)$ decouple in the energy eigenbasis. In the Markovian limit, tunneling rates and bath expectation values are all evaluated at the transition frequencies of the system. Since the SET Coulomb shift depends on the position of the charge qubit electron, this introduces for finite SET bias effective tunneling rates $\Gamma_{a}$ and $\Gamma_{a}^{\prime}$ for lower and upper electron position, respectively, such that the (time and ensemble averaged) SET current comprises damped oscillations for $\Gamma_{a}^{\prime} \neq \Gamma_{a}$, see Fig. 1(d). These are caused by the qubit evolution [Fig. 1(e)]. With the SET occupation $n(t)=\operatorname{Tr}[\hat{n} \rho(t)]$ and the Bloch vector components conditioned on whether the SET is occupied, $S_{1}^{\alpha}(t) \equiv \operatorname{Tr}\left[\left(\hat{n} \otimes \hat{\sigma}_{\alpha}\right) \rho(t)\right]$ and $S_{0}^{\alpha}(t) \equiv \operatorname{Tr}\left\{\left[(1-\hat{n}) \otimes \hat{\sigma}_{\alpha}\right] \rho(t)\right\}(\alpha=x, y, z)$ respectively, such that $S^{\alpha}(t)=S_{0}^{\alpha}(t)+S_{1}^{\alpha}(t)$, the quantum master equation has the following convenient vector form (omit- 


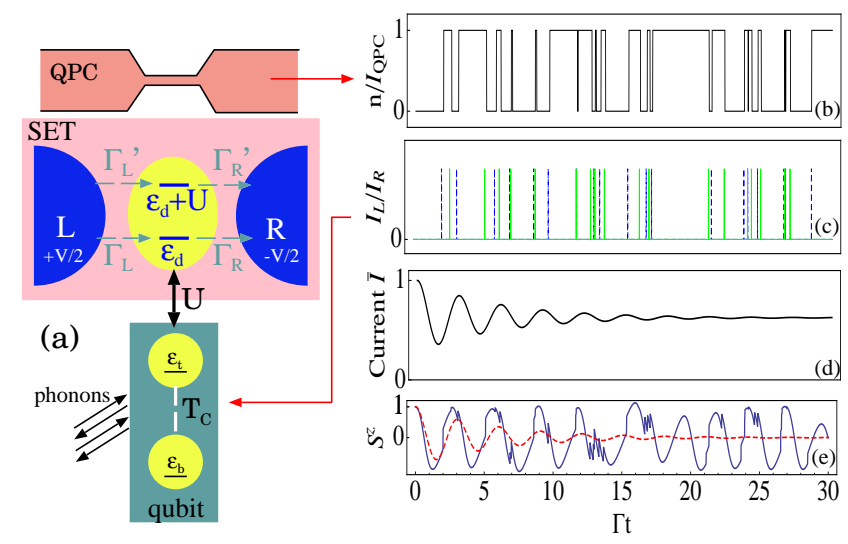

FIG. 1: (color online). (a) Sketch of the device: charge qubit and single-electron transistor (SET) as capacitively coupled detector; a quantum point contact (QPC) is attached to the SET to resolve $I_{L}(t)$ and $I_{R}(t)$ for the closed-loop feedback. (b) Single realization of the SET occupation $n(t)$ measured by the QPC current $I_{\mathrm{QPC}}(t)$. (c) Single realizations of SET currents $I_{L}(t)$ (blue, solid) and $I_{R}(t)$ (dashed, green). (d) SET current $\langle\bar{I}\rangle(t)$, ensemble averaged $\langle$. $\rangle$ and time averaged $\bar{I}=\frac{1}{\tau} \int_{t}^{t+\tau} I\left(t^{\prime}\right) d t^{\prime}$ with $I(t)=\frac{1}{2}\left[I_{L}(t)+I_{R}(t)\right]$, exhibits damped oscillations caused by the coupling to the qubit. (e) Bloch vector component $S^{z}(t)$ (qubit occupation): ensemble average (red, dashed) shows oscillations damped by the presence of the SET, single realization (blue, solid) exhibits free evolution interrupted whenever an electron jumps at the SET. Parameters: $\Gamma^{\prime} / \Gamma=4, T_{c} / \Gamma=1, \varepsilon=0, U / \Gamma=0.5$, measurement time $\Gamma \tau=0.01$, initial state: $S^{z}(0)=1, n(0)=0$.

ting the time dependence):

$$
\begin{aligned}
\dot{n} & =\gamma_{L}^{+}-\gamma^{+} n-\gamma_{L}^{-} S_{0}^{z}+\gamma_{R}^{-} S_{1}^{z}, \\
\dot{\mathbf{S}}_{0} & =\mathbf{A}_{0} \times \mathbf{S}_{0}+\mathcal{D}_{L} \mathbf{S}_{0}+\mathcal{B}_{R} \mathbf{S}_{1}+\mathbf{n}_{0} n+\left(\eta_{r}, 0, \gamma_{L}^{-}\right)^{T}, \\
\dot{\mathbf{S}}_{1} & =\mathbf{A}_{1} \times \mathbf{S}_{1}+\mathcal{D}_{R} \mathbf{S}_{1}+\mathcal{B}_{L} \mathbf{S}_{0}+\mathbf{n}_{1} n-\left(0,0, \gamma_{L}^{-}\right)^{T},(2)
\end{aligned}
$$

with $\quad \mathbf{S}_{i}(t) \equiv\left(S_{i}^{x}(t), S_{i}^{y}(t), S_{i}^{z}(t)\right)^{T}, \quad \gamma_{a}^{ \pm} \equiv \frac{1}{2}\left(\Gamma_{a} \pm \Gamma_{a}^{\prime}\right)$, $\gamma^{ \pm} \equiv \gamma_{L}^{ \pm}+\gamma_{R}^{ \pm}, \mathbf{n}_{0}=-\mathbf{n}_{1} \equiv-\left(\eta_{r}, 0, \gamma^{-}\right)^{T}$, and

$$
\begin{aligned}
\mathcal{D}_{a} & \equiv\left(\begin{array}{ccc}
-\gamma_{a}^{+}-\eta_{p} & 0 & \eta_{+}+\eta_{-} \\
0 & -\gamma_{a}^{+}-\eta_{p} & 0 \\
0 & 0 & -\gamma_{a}^{+}
\end{array}\right), \\
\mathcal{B}_{a} & \equiv \operatorname{Diag}\left(\sqrt{\Gamma_{a} \Gamma_{a}^{\prime}}, \sqrt{\Gamma_{a} \Gamma_{a}^{\prime}}, \gamma_{a}^{+}\right)
\end{aligned}
$$

with the phonon rates $\eta_{p}$ and $\eta_{r} \equiv \eta_{+}-\eta_{-}$explicitly defined in Ref. [10]. For zero qubit bias $(\varepsilon=0)$ they are $\eta_{r}=g \pi T_{c} e^{-2 T_{c} / \omega_{c}}$ and $\eta_{p}=\eta_{r} \operatorname{coth}\left(\beta T_{c}\right)$ with the dimensionless coupling strength $g$, the cut-off frequency $\omega_{c}$, and the inverse temperature $\beta$. The free dynamics of $\mathbf{S}_{j}(t)$ in Eqs. (2) can be considered as a precession around vector $\mathbf{A}_{j} \equiv-\left(2 T_{c}, 0, \varepsilon+j U\right)^{T}$ with frequency $\Delta \equiv \sqrt{\varepsilon^{2}+4 T_{c}^{2}}(j=0)$ or $\Delta^{\prime} \equiv \sqrt{(\varepsilon+U)^{2}+4 T_{c}^{2}}(j=$ $1)$, respectively. The effect of dissipation is contained in $\mathcal{D}_{a}$ : damping of all Bloch vector components by the presence of the SET with $\gamma_{L}^{+}$or $\gamma_{R}^{+}$and by the electronphonon coupling which leads to an additional decay and

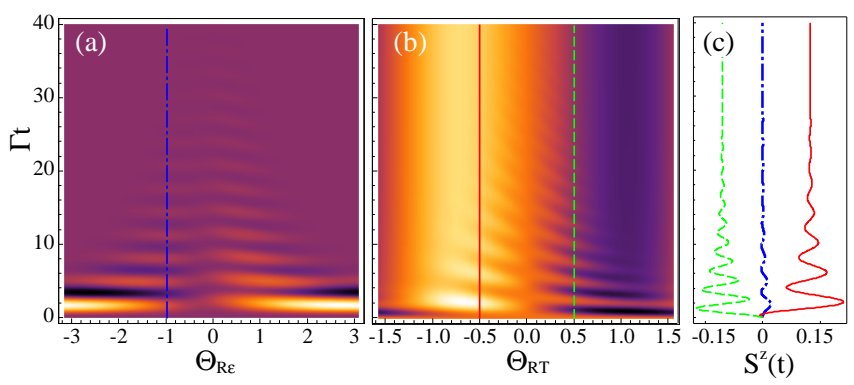

FIG. 2: (color online). Bloch vector component $S^{z}(t)$ vs. time $\Gamma t$ and feedback parameter $\Theta_{R \varepsilon}$ (a) and $\Theta_{R T}$ (b); (c) $S^{z}(t)$ cross-sections at $\Theta_{R \varepsilon}=-1$ (blue, dash-dotted), $\Theta_{R T}=$ -0.5 (red, full), 0.5 (green, dashed). Parameters: $\varepsilon=0$, $U / \Gamma=0.5, \Gamma^{\prime} / \Gamma=3, T_{c} / \Gamma=1$. Initial state is the complete statistical mixture with empty SET. With feedback damped qubit oscillations can be induced which would not occur for vanishing SET-qubit coupling $\Gamma=\Gamma^{\prime}$.

scattering of the $S_{i}^{x / y}(t)$. The conditioned Bloch vectors $\mathbf{S}_{j}(t)$ are mutually coupled via the diagonal matrices $\mathcal{B}_{L / R}$, i.e., by electron hopping onto the SET and out of it, respectively. We provide an analytical solution in the online supplement [11]. The SET occupation couples to the qubit and is not affected by its dynamics for $\Gamma_{a}=\Gamma_{a}^{\prime}$. We note that the Coulomb interaction $U$ enters Eqs. (2) implicitly in $\Gamma^{\prime}$ and explicitly in $\mathbf{A}_{1}$. Whenever we set $U=0$ in the following we do not exclude $\Gamma^{\prime} \neq \Gamma$ in order to maintain the coupling but rather neglect the physical back-action on the qubit.

Detector counting statistics and active measurement. The information about the number of charges $n_{a}$ transferred through a multi-lead conductor (our SET detector) at the $a$-th lead can be gained by the help of $n_{a^{-}}$ resolved quantum master equations (see e.g. Ref. [12] for detailed discussions). The unraveled Fourier-transformed master equation for unidirectional transport is $\dot{\rho}=\left[\mathcal{L}_{0}+\right.$ $\left.\sum_{a} e^{i \chi_{a}} \mathcal{J}_{a}\right] \rho \equiv \mathcal{L}\left(\left\{\chi_{a}\right\}\right) \rho$, where the $\mathcal{J}_{a}\left(\mathcal{L}_{0}\right)$ denote jump (non-jump) operators and $\chi_{a}$ are the counting fields and the system state $\rho$ is not actively controlled (passive measurement). In contrast, when active control operations on the system depending on the measurement outcomes are performed after single jump events, the Fourier-transformed master equation generalizes to

$$
\frac{d}{d t} \rho\left(\left\{\chi_{a}\right\}, t\right)=\left[\mathcal{L}_{0}+\sum_{a} e^{i \chi_{a}} e^{\hat{K}_{a}} \mathcal{J}_{a}\right] \rho\left(\left\{\chi_{a}\right\}, t\right)
$$

where the $\hat{\mathcal{K}}_{a}$ describe the control operation [1]. In contrast to passive measurements, in an active (control) measurement the (scalar) counting fields $\chi_{a}$ are thus upgraded to superoperators, $i \chi_{a} \rightarrow i \chi_{a}+\hat{\mathcal{K}}_{a}$.

Feedback scheme. - We choose the feedback of the SET current acting on $T_{c}$ or/and on $\varepsilon$ which are accessible in present-days setups by appropriate gate voltages. The corresponding feedback Hamiltonian is defined by 
$H_{\mathrm{fb}}=\sum_{a=L / R} I_{a}(t) h_{a}$ with $h_{a} \equiv \Theta_{a \varepsilon} \hat{\sigma}_{z}+\Theta_{a T} \hat{\sigma}_{x}$ and the feedback parameters $\Theta_{a T}$ and $\Theta_{a \varepsilon}$. Experimentally, single jump events $I_{L / R}(t)$ may neither be resolved nor measured independently in the SET circuit. Therefore, we propose an additional quantum-point contact (QPC) weakly attached to the SET as sketched in Fig. 1(a). Its telegraph-like current signal $I_{\mathrm{QPC}}(t)$ provides the occupation of the SET [Fig. 1(b)]. For $\Gamma_{a}^{\prime}>\Gamma_{a}$ the upwards/downwards steps are related to single jumps into/out of the SET and, consequently, $I_{L}(t) / I_{R}(t)$ are trains of $\delta$-kicks [Fig. T(c)]. The superoperators $\hat{\mathcal{K}}_{L / R}$ in the feedback master equation (4) are then given by $\hat{\mathcal{K}}_{a} \rho=-i\left[h_{a}, \rho\right]$. With feedback into $\varepsilon / T_{c}$, the diagonal matrices $\mathcal{B}_{L / R}$ (3) become rotation matrices around the qubit $x / z$-axis [11], so that whenever an electron jumps into/out of the SET the qubit state is rotated by the corresponding angle.

Feedback-induced qubit oscillations. - As a remarkable effect of this Markovian feedback, we find damped qubit oscillations even when the qubit is initially prepared in a completely mixed state (i.e., in the origin of the Bloch sphere: $\mathbf{S}=\mathbf{0}$ ). For low electron-phonon coupling without feedback [1] or for vanishing SET-qubit coupling $\left(\Gamma^{\prime}=\Gamma\right.$, i.e., the qubit kicked by a random sequence of $\delta$-pulses) the qubit would remain completely mixed. Fig. 2 shows the oscillations in $S^{z}(t)$ for a feedback from $I_{R}(t)\left[I_{L}(t)\right.$ provides similar results $]$ into (a) $\varepsilon$ and (b) $T_{c}$. The feedback into $\varepsilon$ [Fig. 2(a)] induces oscillations with the frequency $\omega_{Q}=\frac{1}{4} \sqrt{\left(8 T_{c}\right)^{2}-\gamma_{S}^{2}}(\varepsilon=0)$ for $8 T_{c}>\gamma_{S}$ where $\gamma_{S}=\frac{1}{2}\left(\sqrt{\Gamma}-\sqrt{\Gamma^{\prime}}\right)^{2}$ is the damping due to SET-qubit coupling [11] and additional feedback damping which increases with $\Theta_{R \varepsilon}$. At large times, the qubit state is again completely mixed. In contrast, for the $T_{c}$-feedback [Fig. 2(b)] a frequency shift towards higher(lower) frequencies for $\Theta_{R T}>(<) 0$ occurs, and in the long-term limit a localization $\left[S^{z}(t \rightarrow \infty) \neq 0\right]$ takes place. The transient oscillations of the qubit occupation [Fig. 2(c)] can be directly measured as damped current oscillations [compare Figs. 1(d) and (e)].

Noise spectrum. - The total noise spectrum of the SET current is computed by (Ramo-Shockley theorem) $S(\omega)=\frac{1}{4} \sum_{a, b=L, R} S_{a b}(\omega)$ for a symmetric SET with $S_{a b}(\omega)=\operatorname{Tr}\left\{\left[\mathcal{J}_{a b}+\mathcal{J}_{a} \Omega(\omega) \mathcal{J}_{b}+\mathcal{J}_{a} \Omega(-\omega) \mathcal{J}_{b}\right] \bar{\rho}\right\} \quad$ where $\Omega(\omega)=[-i \omega \mathbb{1}-\mathcal{L}(0,0)]^{-1}$, steady state $\bar{\rho}$ with $\mathcal{L}(0,0) \bar{\rho}=$ 0 , and the jump operators $\left.\mathcal{J}_{a} \equiv \partial_{i \chi_{a}} \mathcal{L}\left(\chi_{L}, \chi_{R}\right)\right|_{(0,0)}$, $\left.\mathcal{J}_{a, b} \equiv \partial_{i \chi_{a}} \partial_{i \chi_{b}} \mathcal{L}\left(\chi_{L}, \chi_{R}\right)\right|_{(0,0)}$. The spectrum provides detailed information on the qubit dynamics such as oscillation frequencies and relaxation rates. Without feedback, $S(\omega)$ shows peaks at the bare qubit oscillation frequency $\Delta$ (for $\gamma_{S} \ll T_{c}$ ) and at the shifted frequency due to Coulomb interaction $\Delta^{\prime}$ for nonvanishing and weak SET-qubit coupling $\Gamma^{\prime} / \Gamma \neq 1$, see inset of Fig. 3(a). We can further use it to monitor the effect of feedback on the qubit dynamics, as shown in Fig. B(a). The most interesting feedback scenario is when both $I_{L}(t)$ and $I_{R}(t)$
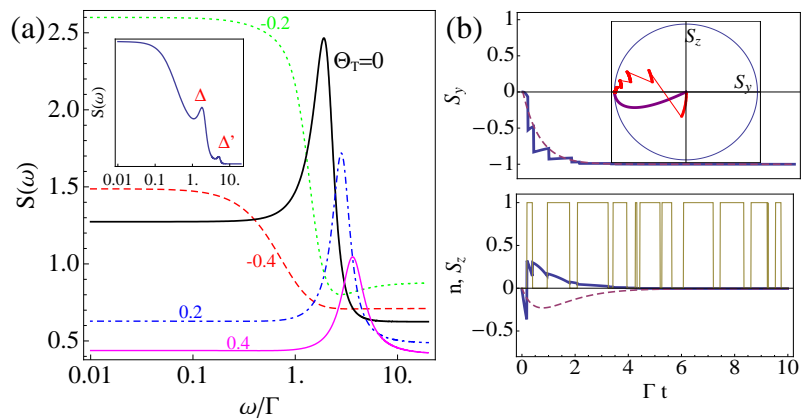

FIG. 3: (color online). (a) SET noise spectrum $S(\omega)$ vs. $\omega$ for various feedback parameter $\Theta_{T}=\Theta_{L T}=\Theta_{R T}=-0.4$ (dashed, red), -0.2 (dotted, green), 0 (thick solid, black), 0.2 (dash-dotted, blue), 0.4 (thin solid, violet); (Inset) $S(\omega)$ for $\Theta_{T}=0, \Gamma^{\prime} / \Gamma=1.5, U / \Gamma=5$. (b) Single realization of Bloch vector components $S^{y, z}(t)$ and SET occupation $n(t)$ vs. time $\Gamma t$ with initially mixed state $\mathbf{S}=0$; ensemble averaged quantities (dashed lines); (Inset) Single realizations in the Bloch sphere; measurement time $\Gamma \tau=0.1$, feedback parameter: $\Theta_{T}=-0.45$. Parameters: $\varepsilon=0, U / \Gamma=1, \Gamma^{\prime} / \Gamma=9$, $T_{c} / \Gamma=1$.

are simultaneously coupled back into $T_{c}$. This yields a frequency shift linear in $\Theta_{T} \equiv \Theta_{L T}=\Theta_{R T}$ [compare Fig. 2(b)] of the $\Delta$-peak which is clearly revealed in $S(\omega)$ [Fig. 3(a)]. Moreover, at low frequencies the noise exhibits a pronounced local minimum w.r.t. $\Theta_{T}$ at small negative values (not shown) which turns out to correspond to the stabilization of pure qubit states.

Generation of pure states - The simulation of the quantum trajectory in Fig. 3(b) indeed confirms that by starting in a complete statistical mixture, the qubit will finally approach the pure state $\frac{1}{\sqrt{2}}(|t\rangle-i|b\rangle)\left(S^{y}=-1\right)$ after a few electron jumps through the SET. In the following we discuss in more detail the stabilization of such pure qubit states. The steady state yields

$$
\bar{n}=\frac{1}{2}, \bar{S}^{y}=\gamma^{-} \frac{4 c T_{c}}{4 T_{c}\left(4 T_{c}+s G\right)+c \gamma^{+}\left(G+2 \eta_{p}\right)},
$$

with $s \equiv \sin \left(2 \Theta_{T}\right), \quad c \equiv 1-\cos \left(2 \Theta_{T}\right)$, and $G \equiv \frac{1}{2}\left(\sqrt{\Gamma}+\sqrt{\Gamma^{\prime}}\right)^{2}$. The $\bar{S}^{x}$-component is solely governed by electron-phonon relaxation [11] (we omit the $\bar{S}^{z}$-component for brevity). We note that the SET occupation is not affected by the feedback due to the high SET bias limit. The steady-state purity $\left[P \equiv \operatorname{Tr}\left(\bar{\rho}^{2}\right)=\right.$ $\left.\sum_{\alpha}\left(\bar{S}^{\alpha}\right)^{2}\right]$, i.e., the length of the Bloch vector for small electron-phonon coupling $\eta_{p} \ll \Gamma$ reads

$$
P=\left(\gamma^{-}\right)^{2} c \frac{c G^{2}+8 T_{c}\left(4 T_{c}+s G\right)}{c \gamma^{+} G+4 T_{c}\left(4 T_{c}+s G\right)^{2}} .
$$

First, for zero feedback $\left(\Theta_{T}=0, c=0\right)$ the purity vanishes as expected. Second, above a critical coupling $\Gamma^{\prime} / \Gamma \geq 1+8 T_{c} / \Gamma$ following from the condition 


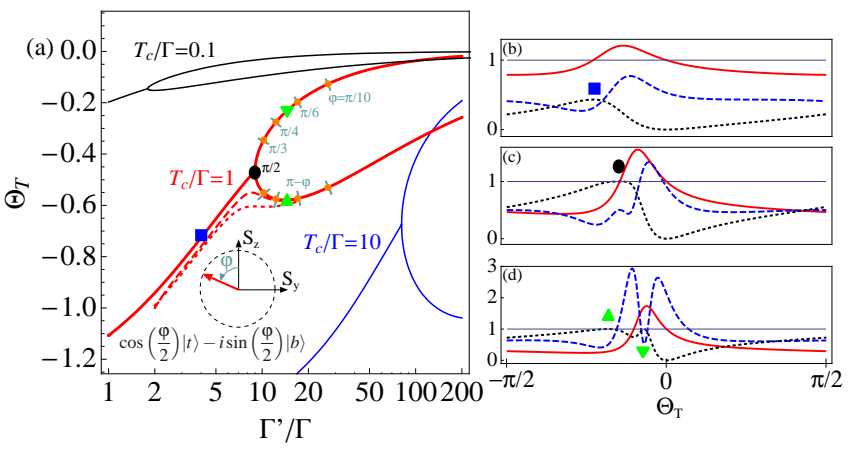

FIG. 4: (color online). (a) Location of purity $P$ maxima in feedback parameter plane $\left(\Theta_{T}, \Gamma^{\prime} / \Gamma\right)$, Eq. (7). Above a critical coupling $\Gamma^{\prime} / \Gamma \geq 1+8 T_{c} / \Gamma$ (bifurcation) the qubit state is pure with $P=1$, otherwise $P<1$. At the bifurcation the qubit state is maximally delocalized with $S^{y}=-1(\varphi=\pi / 2)$ and above $S^{z} \neq 0$ with $0<\varphi<\pi, \varphi \neq \pi / 2$ holds. The bifurcation becomes blurred and the complete delocalization is diminished for $U / \Gamma=0.5$ (dashed line) and/or $\eta_{r} / \Gamma=$ $0.1, \beta T_{c}=1$ (dotted line). (b)-(d) Normalized steady-state Current $I / I_{0}$ with $I_{0} \equiv \frac{\Gamma+\Gamma^{\prime}}{8}$ (red, solid curves), Fano factor $F=S(0) /(2 I)$ (blue, dashed curves), purity $P=\operatorname{Tr}\left(\rho^{2}\right)$ (black, dotted curves) vs. $\Theta_{T}$. Parameters: $\varepsilon=U=0$, $T_{c} / \Gamma=1, \Gamma^{\prime} / \Gamma=4$ (b), 9 (c), 15 (d).

$B \equiv \sqrt{\left(\gamma^{-}\right)^{2}-16 T_{c}^{2}} \geq 0$, two maxima of $P$ exist:

$\cos ^{2}\left(\Theta_{T}^{ \pm}\right)=\frac{G\left[\left(\gamma^{+}\right)^{2}+D+12 T_{c}^{2}\right]-2 \gamma^{+} D \pm 8 T_{c}^{2} B}{G\left(\Gamma \Gamma^{\prime}+16 T_{c}^{2}\right)},(7)$

with $D \equiv G^{2}+4 T_{c}^{2}$ where the stationary qubit state has purity $P=1$. Below the bifurcation $\left(B^{2}<0\right)$ the state is partially mixed $(P<1)$ and

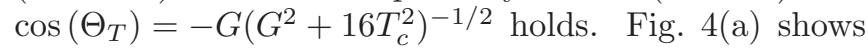
this bifurcation behavior of the maximal purity for various $T_{c}$. The bifurcation point $(B=0)$ corresponds to the completely delocalized state with $S^{y}=-1(\varphi=\pi / 2)$ The sign of $S^{y}$ is determined by $\gamma^{-}$, compare Eq. (5). In the upper(lower) branch the pure qubit state becomes increasingly localized with $S^{z}>0\left(S^{z}<0\right)$. The effect of finite $U$ and the phononic environment onto the steady-state purity is essentially a blurring of the bifurcation in Fig. 4(a) (dashed and dotted curves). A complete delocalization $\left(S^{y}=-1\right)$ as for the noninteracting case hence cannot be obtained here anymore. Nevertheless, asymptotic purification with increasing localization can be realized in the limit of large SET-qubit coupling.

Steady-state SET transport. - Without feedback we obtain the steady-state current $I_{0} / e=\left(\Gamma+\Gamma^{\prime}\right) / 8$ and the Fano factor $F \equiv \frac{S(0)}{2 I}=\frac{1}{2}+2 \frac{\left(\gamma_{S}+2 \eta_{p}\right)\left(\Gamma-\Gamma^{\prime}\right)^{2}}{\left(8 T_{c}\right)^{2}\left(\Gamma+\Gamma^{\prime}\right)}$. The SET current is not affected by the phonons, whereas the noise carries information about the interaction of the qubit with the SET detector $\gamma_{S}$ and the phononic environment $\eta_{p}$. The Fano factor is one-half for vanishing SET-qubit coupling $\left(\Gamma=\Gamma^{\prime}\right)$ and diverges for large SET-qubit coupling $\left(\Gamma^{\prime} / \Gamma \gg 1\right)$ which results from the bistable cur- rent behavior (quantum Zeno effect). It also diverges for $T_{c} \rightarrow 0$ since the two subspaces decouple in this limit. Figs. 4(b)-(d) present the $\Theta_{T}$-dependencies of the steadystate current and the Fano factor at various detectorqubit couplings $\Gamma^{\prime} / \Gamma$. The current, in principle, follows the electron localization in the qubit. At the critical coupling [Fig. 4 (c)] when $P=1$ the qubit state is maximally delocalized so that the current approaches its no-feedback value $I_{0}$ and the Fano factor displays a local maximum. For larger couplings [Fig. 4(d)] the purity maximum in the upper branch is accompanied with a current maximum and a Fano factor minimum. Hence, the degree of the qubit state purity can be very accurately monitored by varying the feedback parameter $\Theta_{T}$.

Conclusions. - We have presented a simple way to stabilize exact pure states and generate charge oscillations in an individual charge qubit by applying an electronic feedback loop. Even for moderate detector back-action and coupling to a dissipative environment (e.g. phonons) an asymptotic purification can be achieved. We note that in contrast to ground state relaxation just by cooling and strong electron-phonon coupling our method also works at high temperatures and, in principle, arbitrary pure states can be produced. The detector current and its noise spectrum are shown to be sensitive monitors for the effect of feedback.

Financial support by the DFG (BR 1528/5, BR 1528/8, SCHA 1646/2-1, and SFB 910) and helpful discussions with F. Haupt, K. Mosshammer and C. Pöltl are gratefully acknowledged.

* Electronic address: gerold.kiesslich@tu-berlin.de

[1] H. M. Wiseman and G. J. Milburn, Quantum Measurement and Control (Cambridge University Press, Cambridge, 2010).

[2] T. Fujisawa et al., Science 112, 1634 (2006); S. Gustavsson et al., Phys. Rev. Lett. 96, 076605 (2006).

[3] K. D. Petersson et al., Phys. Rev. Lett. 105, 246804 (2010).

[4] S. A. Gurvitz, Phys. Rev. B 56, 15215 (1997); A. Shnirman and G. Schön, Phys. Rev. B 57, 15400 (1998); H.S. Goan et al., Phys. Rev. B 63, 125326 (2001); A. N. Korotkov, Phys. Rev. B 63, 115403 (2001); S. Pilgram and M. Büttiker, Phys. Rev. Lett. 89, 200401 (2002); N. P. Oxtoby et al., Phys. Rev. B 74, 045328 (2006); I. Neder and F. Marquardt, New J. Phys. 9, 112 (2007); S. A. Gurvitz and D. Mozyrsky, Phys. Rev. B 77, 075325 (2008).

[5] Handbook of chaos control, edited by E. Schöll and H. G. Schuster, (Wiley-VCH, Weinheim, 2008).

[6] R. Ruskov and A. N. Korotkov, Phys. Rev. B 66, 041401 (2002).

[7] S.-K. Wang et al., Phys. Rev. B 75, 155304 (2007).

[8] A. N. Korotkov, Phys. Rev. B 71, 201305 (2005).

[9] H. Bluhm et al., Phys. Rev. Lett. 105, 216803 (2010).

[10] G. Kießlich et al., Phys. Rev. Lett. 99, 206602 (2007).

[11] See supplementary material.

[12] G. Schaller et al., Phys. Rev. B 80, 245107 (2009). 


\title{
Supplementary Material: \\ Charge Qubit Purification by an Electronic Feedback Loop
}

\author{
G. Kießlich $*$ G. Schaller, C. Emary, and T. Brandes \\ Institut für Theoretische Physik, Hardenbergstraße 36, \\ Technische Universität Berlin, D-10623 Berlin, Germany
}

Equation numbers in the main text are referred to by an asterisk.

\section{QUBIT DYNAMICS WITHOUT FEEDBACK}

We rewrite Eqns. $\left(2^{*}\right)$ as vector equation $\dot{\mathbf{x}}=\mathcal{C} \cdot \mathbf{x}+\mathbf{v}$ with

$$
\begin{aligned}
\mathcal{C} & \equiv\left(\begin{array}{ccc}
-\gamma^{+} & -\gamma_{L}^{-} \mathbf{e}_{z}^{T} & \gamma_{R}^{-} \mathbf{e}_{z}^{T} \\
\mathbf{n}_{0} & \mathcal{A}_{0}+\mathcal{D}_{L} & \mathcal{B}_{R} \\
\mathbf{n}_{1} & \mathcal{B}_{L} & \mathcal{A}_{1}+\mathcal{D}_{R}
\end{array}\right), \\
\mathbf{x} & \equiv\left(n, \mathbf{S}_{0}, \mathbf{S}_{1}\right)^{T}, \quad \mathbf{v} \equiv\left(\gamma_{L}^{+}, \gamma_{r}^{+} \mathbf{e}_{x}+\gamma_{L}^{-} \mathbf{e}_{z},-\gamma_{L}^{-} \mathbf{e}_{z}\right)^{T} .
\end{aligned}
$$

For its solution we use the Laplace transform $\tilde{\mathbf{x}}(z)=[z \mathbb{1}-\mathcal{C}]^{-1} \cdot\left(\mathbf{x}_{0}+\mathbf{v} / z\right)$ with the initial state $\mathbf{x}_{0} \equiv\left(n_{0}, S_{0}^{x 0}, S_{0}^{y 0}, S_{0}^{z 0}, S_{1}^{x 0}, S_{1}^{y^{0}}, S_{1}^{z 0}\right)^{T}$.

For symmetric coupling $\left(\Gamma / 2 \equiv \Gamma_{L}=\Gamma_{R}, \Gamma^{\prime} / 2 \equiv \Gamma_{L}^{\prime}=\Gamma_{R}^{\prime}\right)$ and $\varepsilon=U=0$ we find

$$
\begin{aligned}
\tilde{n}(z) & =\frac{A_{1}\left(z, n_{0}\right)+z A_{2}(z)+\left(\gamma^{+}+2 n_{0} z\right) A_{3}(z)}{2 z\left[A_{1}(z, 1)+\left(\gamma^{+}+z\right) A_{3}(z)\right]}, \\
\tilde{S}^{x}(z) & =\frac{2}{g(z)} S^{x 0}, \quad \tilde{S}^{y}(z)=2 \frac{2 T_{c} S^{z 0}+z S^{y 0}}{8 T_{c}^{2}+z g(z)}, \\
\tilde{S}^{z}(z) & =\frac{-4 T_{c} S^{y 0}+g(z) S^{z 0}}{8 T_{c}^{2}+z g(z)},
\end{aligned}
$$

with $\quad A_{1}\left(z, n_{0}\right) \equiv \sqrt{\Gamma \Gamma^{\prime}}\left[\Gamma \Gamma^{\prime}+2 n_{0} z\left(\gamma^{+}+z\right)\right]-\left(\gamma^{-}\right)^{2}\left(\gamma^{+}+2 z\right), \quad A_{2}(z) \equiv \sqrt{\Gamma \Gamma^{\prime}} \gamma^{+}-\gamma^{-}\left[g(z) S^{z-}-4 T_{c} S^{y-}\right]$, $A_{3}(z) \equiv 8 T_{c}^{2}+\left(\gamma^{+}+z\right)\left(\gamma^{+}+2 z\right), S^{y / z-} \equiv S_{0}^{y / z 0}-S_{1}^{y / z 0}$, and $g(z) \equiv \gamma^{+}-\sqrt{\Gamma \Gamma^{\prime}}+2 z$.

The inverse Laplace transform for $\varepsilon=U=0$ yields

$$
\begin{aligned}
S^{x}(t) & =\frac{2 \eta_{r}\left(1-e^{-\frac{1}{2} \gamma_{D} t}\right)}{\gamma_{S}+2 \eta_{r} \operatorname{coth}\left(\beta T_{c}\right)}+e^{-\frac{1}{2} \gamma_{D} t} S^{x 0}, \\
S^{y / z}(t) & =e^{-\frac{1}{4} \gamma_{D} t}\left[S^{y / z 0} \cosh \left(\omega_{Q} t\right) \mp \frac{\gamma_{D} S^{y / z 0}-8 T_{c} S^{z / y 0}}{4 \omega_{Q}} \sinh \left(\omega_{Q} t\right)\right],
\end{aligned}
$$

with $\omega_{Q} \equiv \frac{1}{4} \sqrt{\gamma_{D}^{2}-\left(8 T_{c}\right)^{2}}$ (for $\gamma_{D} \ll 8 T_{c}$ damped qubit oscillations occur.) and $\gamma_{D} \equiv \gamma_{S}+2 \eta_{p}$ where $\gamma_{S} \equiv$ $\frac{1}{2}\left(\sqrt{\Gamma}-\sqrt{\Gamma^{\prime}}\right)^{2}$ is the decoherence rate due to the SET-qubit coupling which was also found for a qubit coupled to a QPC in Ref. [1] where the respective rates are given by the QPC transmissions $T$ and $T^{\prime}$. For $U>0$ the expressions become rather cumbersome such that the decay can be non-exponential (see e.g. Ref. 2] and discussions therein). The steady state can be readily obtained by $\lim _{z \rightarrow 0^{+}} z \tilde{\mathbf{x}}(z)$ and yields (also for $\varepsilon, U \neq 0$ )

$$
\bar{n}=\frac{1}{2}, \quad \overline{\mathbf{S}}=\left(\frac{2 \eta_{r}}{\gamma_{S}+2 \eta_{r} \operatorname{coth}\left(\beta T_{c}\right)}, 0,0\right)^{T}
$$

with $\eta_{r}=g \pi T_{c} e^{-2 T_{c} / \omega_{c}}$ being the phonon relaxation rate where $g$ is the dimensionless electron-phonon coupling strength and $\omega_{c}$ is a cut-off frequency [3]. For zero phonon temperature $(\beta \rightarrow \infty)$ the $x$-component becomes $y /(1+y)\left(y \equiv 2 \eta_{r} / \gamma_{S}\right)$ also discussed in Ref. [4]; the qubit relaxes into a pure state for $y \gg 1$ (qubit ground state). For large temperatures $(\beta \rightarrow 0)$ the qubit will behave as statistical mixture. 


\section{FEEDBACK-MODIFIED EQUATIONS OF MOTION}

The feedback scheme modifies Eqns. ( $\left.2^{*}\right)$ such that $\mathcal{B}_{a} \rightarrow \mathcal{B}_{a}^{f}, \mathbf{n}_{0} \rightarrow \mathbf{n}_{0}^{f}, \mathbf{n}_{1} \rightarrow \mathbf{n}_{1}^{f}$, and $\mathbf{v} \rightarrow \mathbf{v}^{f}$. For $\Theta_{a T}=0$ we find $n_{0}^{f}=n_{0}, n_{1}^{f}=n_{1}, \mathbf{v}^{f}=\mathbf{v}$, and

$$
\mathcal{B}_{a}^{f}=\left(\begin{array}{ccc}
\cos \left(\Theta_{a \varepsilon}\right) \sqrt{\Gamma_{a} \Gamma_{a}^{\prime}} & \sin \left(\Theta_{a \varepsilon}\right) \sqrt{\Gamma_{a} \Gamma_{a}^{\prime}} & 0 \\
-\sin \left(\Theta_{a \varepsilon}\right) \sqrt{\Gamma_{a} \Gamma_{a}^{\prime}} & \cos \left(\Theta_{a \varepsilon}\right) \sqrt{\Gamma_{a} \Gamma_{a}^{\prime}} & 0 \\
0 & 0 & \gamma_{R}^{+}
\end{array}\right),
$$

which represents a rotation of the Bloch vector around the $z$-axis with angle $\Theta_{a \varepsilon}$. Respectively for $\Theta_{a \varepsilon}=0$ the matrix $\mathcal{B}_{a}^{f}$ rotates the Bloch vector around the $x$-axis with angle $\Theta_{a T}$ :

$$
\begin{aligned}
& \mathcal{B}_{a}^{f}=\left(\begin{array}{ccc}
\sqrt{\Gamma_{a} \Gamma_{a}^{\prime}} & 0 & 0 \\
0 & \cos \left(2 \Theta_{a T}\right) \sqrt{\Gamma_{a} \Gamma_{a}^{\prime}} & \sin \left(2 \Theta_{a T}\right) \gamma_{a}^{+} \\
0 & -\sin \left(2 \Theta_{a T}\right) \sqrt{\Gamma_{a} \Gamma_{a}^{\prime}} & \cos \left(2 \Theta_{a T}\right) \gamma_{a}^{+}
\end{array}\right), \\
& n_{0}^{f}=-\gamma_{R}^{-}\left(0, \sin \left(2 \Theta_{R T}\right), \cos \left(2 \Theta_{R T}\right)+\frac{\gamma_{L}^{-}}{\gamma_{R}^{-}}\right)^{T}, \\
& n_{1}^{f}=\gamma_{L}^{-}\left(0, \sin \left(2 \Theta_{L T}\right), \cos \left(2 \Theta_{L T}\right)+\frac{\gamma_{R}^{-}}{\gamma_{L}^{-}}\right)^{T} \text {, } \\
& \mathbf{v}^{f}=\left(\gamma_{L}^{+}, \gamma_{L}^{-} \mathbf{e}_{z},-\gamma_{L}^{-}\left[\sin \left(2 \Theta_{L T}\right) \mathbf{e}_{y}+\cos \left(2 \Theta_{L T}\right) \mathbf{e}_{z}\right]\right)^{T} .
\end{aligned}
$$

For symmetric coupling and $\varepsilon=U=0$ the $x$-components decouple, and we can truncate the dynamical system to 5 equations:

$$
\mathcal{C}^{f}=\left(\begin{array}{c|cc|cc}
-\gamma^{+} & 0 & -\frac{\gamma^{-}}{2} & 0 & \frac{\gamma^{-}}{2} \\
\hline-\frac{\gamma^{-}}{2} s_{R} & -\frac{\gamma^{+}}{2}-\eta_{p} & 2 T_{c} & \frac{\sqrt{\Gamma \Gamma^{\prime}}}{2} c_{R} & \frac{\gamma^{+}}{2} s_{R} \\
-\frac{\gamma^{-}}{2}\left(1+c_{R}\right) & -2 T_{c} & -\frac{\gamma^{+}}{2} & -\frac{\sqrt{\Gamma \Gamma^{\prime}}}{2} s_{R} & \frac{\gamma^{+}}{2} c_{R} \\
\hline \frac{\gamma^{-}}{2} s_{L} & \frac{\sqrt{\Gamma \Gamma^{\prime}}}{2} c_{L} & \frac{\gamma^{+}}{2} s_{L} & -\frac{\gamma^{+}}{2}-\eta_{p} & 2 T_{c} \\
\frac{\gamma^{-}}{2}\left(1+c_{L}\right) & -\frac{\sqrt{\Gamma \Gamma^{\prime}}}{2} s_{L} & \frac{\gamma^{+}}{2} c_{L} & -2 T_{c} & -\frac{\gamma^{+}}{2}
\end{array}\right)
$$

with $s_{a} \equiv \sin \left(2 \Theta_{a T}\right)$ and $c_{a} \equiv \cos \left(2 \Theta_{a T}\right)$.

* Electronic address: gerold.kiesslich@tu-berlin.de

[1] S. A. Gurvitz, Phys. Rev. B 56, 15215 (1997).

[2] C. Emary, Phys. Rev. A 78, 032105 (2008).

[3] T. Brandes and T. Vorrath, Phys. Rev. B 66, 075341 (2002).

[4] S. A. Gurvitz, L. Fedichkin, D. Mozyrsky, and G. P. Berman, Phys. Rev. Lett. 91, 066801 (2003). 